\title{
A comparison of the growth status, level of blood glucose, and lipid metabolism in small for gestational age and appropriate for gestational age girls with central precocious puberty: a retrospective study
}

\author{
Xiao Yu", Xi Yang", Yue Zhao, Xiao-Ping Luo, Yan Liang, Wei Wu, Yan-Qin Ying, Feng Ye, Ling Hou \\ Department of Pediatrics, Tongji Hospital, Tongji Medical College, Huazhong University of Science and Technology, Wuhan, China \\ Contributions: (I) Conception and design: L Hou; (II) Administrative support: XP Luo, Yan Liang, W Wu, YQ Ying, L Hou; (III) Provision of study \\ materials or patients: XP Luo, Y Liang, W Wu, YQ Ying, L Hou; (IV) Collection and assembly of data: X Yu, X Yang, Y Zhao, L Hou; (V) Data \\ analysis and interpretation: X Yu, X Yang, YQ Ying, H Ling; (VI) Manuscript writing: All authors; (VII) Final approval of manuscript: All authors. \\ \#These authors contributed equally to this work. \\ Correspondence to: Ling Hou. Department of Pediatrics, Tongji Hospital, Tongji Medical College, Huazhong University of Science and Technology, \\ NO.1095 Jiefang Avenue, Wuhan 430030, China. Email: houl63258@163.com.
}

\begin{abstract}
Background: To compare the physical development status, level of blood glucose and lipid metabolism in small for gestational age (SGA) and appropriate for gestational age (AGA) groups with central precocious puberty (CPP).
\end{abstract}

Methods: This was a retrospective study. Three hundred and twenty-two girls with CPP were divided into the AGA group (304 cases) and the SGA group (18 cases). Physical index such as height, weight, and body mass index (BMI), as well as sex hormones, adrenal androgens, blood lipid levels, fasting blood glucose, insulin levels, and homeostasis model assessment of insulin resistance (HOMA-IR) were compared between the two groups.

Results: Height, weight, and BMI in the SGA group were lower than those in the AGA group $(\mathrm{P}<0.05)$. The level of LH/FSH, estradiol, testosterone, DHEA and androstenedione had no significant difference between the SGA group and AGA group $(\mathrm{P}>0.05)$. The fasting blood glucose, insulin level, HOMA-IR, high-density lipoprotein (HDL) and the average level of triglycerides were similar between these two groups $(\mathrm{P}>0.05)$. There was a statistically significant difference in total cholesterol and low-density lipoprotein (LDL) between the two groups $(\mathrm{P}<0.05)$. However, the blood lipids and blood glucose in both groups were within the normal range.

Conclusions: The height, weight, BMI, serum cholesterol and LDL of girls in SGA with CPP were significantly lower than that of those girls born AGA.

Keywords: Small for gestational age (SGA); precocious puberty; growth; puberty; metabolic syndrome

Submitted Nov 04, 2020. Accepted for publication Feb 26, 2021.

doi: $10.21037 / \mathrm{tp}-20-366$

View this article at: http://dx.doi.org/10.21037/tp-20-366

\section{Introduction}

Idiopathic central precocious puberty (ICPP) refers to the secondary sexual characteristics and rapid genital development of children (girls: before 8 years old; boy: before 9 years old) (1). The earliest clinical manifestation in girls is usually breast development (thelarche), followed by pubic hair (pub-arche), and then menarche. In boys, the initial clinical sign is testicular enlargement (2). If children with small for gestational age (SGA) cannot recover on their own, they should be treated with growth hormone. In 
ICPP, puberty is advanced and the increase of sex hormone levels can lead to accelerated maturation and early closure of the epiphysis and the space and time for growth are greatly reduced, thus, affecting the adult height of the child and causing social-psychological problems (3). Previous studies reported that SGA maybe a risk factor for ICPP $(4,5)$. It is reported that baby girl, passive smoking, peanut oil consumption and unsalted taste were risk factors for SGA, while folic acid supplementation was protective factor (6). Compared with appropriate for gestational age (AGA) girls, the age of ICPP in SGA is earlier and progresses faster, thus, SGA is at a higher risk of being below the target height. It has been reported that SGA girls gain weight rapidly during early childhood, but maybe have an increased risk of metabolic syndrome in adults (7). A recent study in South Korea showed that compared with AGA girls, SGA girls with precocious puberty have increased bone age and earlier menarche. However, no difference was found in FAH after treatment between these groups (8). Moreover, this article did not compare the metabolism of glucose and lipid between the two groups of children. Previous studies found that girls and adolescents born with SGA maybe suffer from metabolic syndrome $(9,10)$. However, it is still unclear whether SGA adult metabolic syndrome is related to the occurrence of ICPP in childhood.

This study compared the growth and development indicators and glucose and lipid metabolism of SGA and AGA girls in the ICPP and explored the effects of SGA on the growth and development of girls, whether SGA girls have abnormalities in glycolipid metabolism when CPP occurs and whether it is related to adulthood metabolic syndrome in SGA, in order to prevent and treat the related diseases at an early stage.

We present the study in accordance with the STROBE reporting checklist (available at http://dx.doi.org/10.21037/ tp-20-366).

\section{Methods}

\section{Research subjects}

In this retrospective study, girls with ICPP in the Tongji Hospital of Tongji Medical College of Huazhong University of Science and Technology from January 2012 to December 2018 were recruited. The percentile of birth weight of infants was based on the curve of birth weight of newborns of different gestational ages in China, formulated in 2015 (11).

\section{Compliance with ethics guidelines}

This study was conducted in accordance with the declaration of Helsinki (as was revised in 2013). This study had been approved by the Ethics Committee of Tongii Hospital of Tongji Medical College of Huazhong University of Science and Technology (No. TJ-IRB20191011). All participants had signed the informed consent. Written informed consent was obtained from the guardians of the participants.

\section{Inclusion and exclusion criteria}

Inclusion criteria: (I) girls developed secondary sexual characteristics before the age of 8; (II) the bone age exceeds the chronological age by more than 1 year; (III) MRI examination of pituitary gland was performed, excluding the central organic lesions.

The exclusion criteria were as follows: peripheral precocious puberty such as gonadal tumor, adrenal tumor, congenital adrenocortical hyperplasia, application of exogenous estrogen, and McCune-Albright syndrome; Partial sexual precocious puberty such as early development of simple breast, early appearance of simple pubic hair, and simple early menarche; Precocious puberty in patients with primary hypothyroidism; Tumor or space-occupying lesion; Central nervous system infection; Acquired injury; Congenital developmental abnormality and so on. In this study, a total of 508 cases were excluded.

\section{Detection method}

All girls underwent a physical development evaluation: height and weight were measured, body mass index (BMI) was calculated, and breast and pubic hair staging were determined by a physical examination. $\mathrm{X}$-ray examination was used to evaluate the bone age. Pelvic B ultrasound examination of uterus and ovary development; MRI was used to exclude intracranial tumor and other organic lesions. Thyrotropin, free thyroxine, testosterone (T), estradiol (E2), 17-hydroxyprogesterone, dehydroepiandrosterone sulfate (DHEAS), and adrenocorticotropin (ACTH) were determined by immunochemiluminometric assays (ICMAS) to exclude hypothyroidism, gonadal tumor, and congenital adrenocortical hyperplasia and tumor indicators were tested and venous blood was extracted after fasting in the morning from 8:00 to 10:00 after resting for 30 minutes.

GnRH stimulation test (4): intravenous injection of GnRH, $2.5 \mu \mathrm{g} / \mathrm{kg}$ (maximum dose $100 \mu \mathrm{g}$ ); the level 

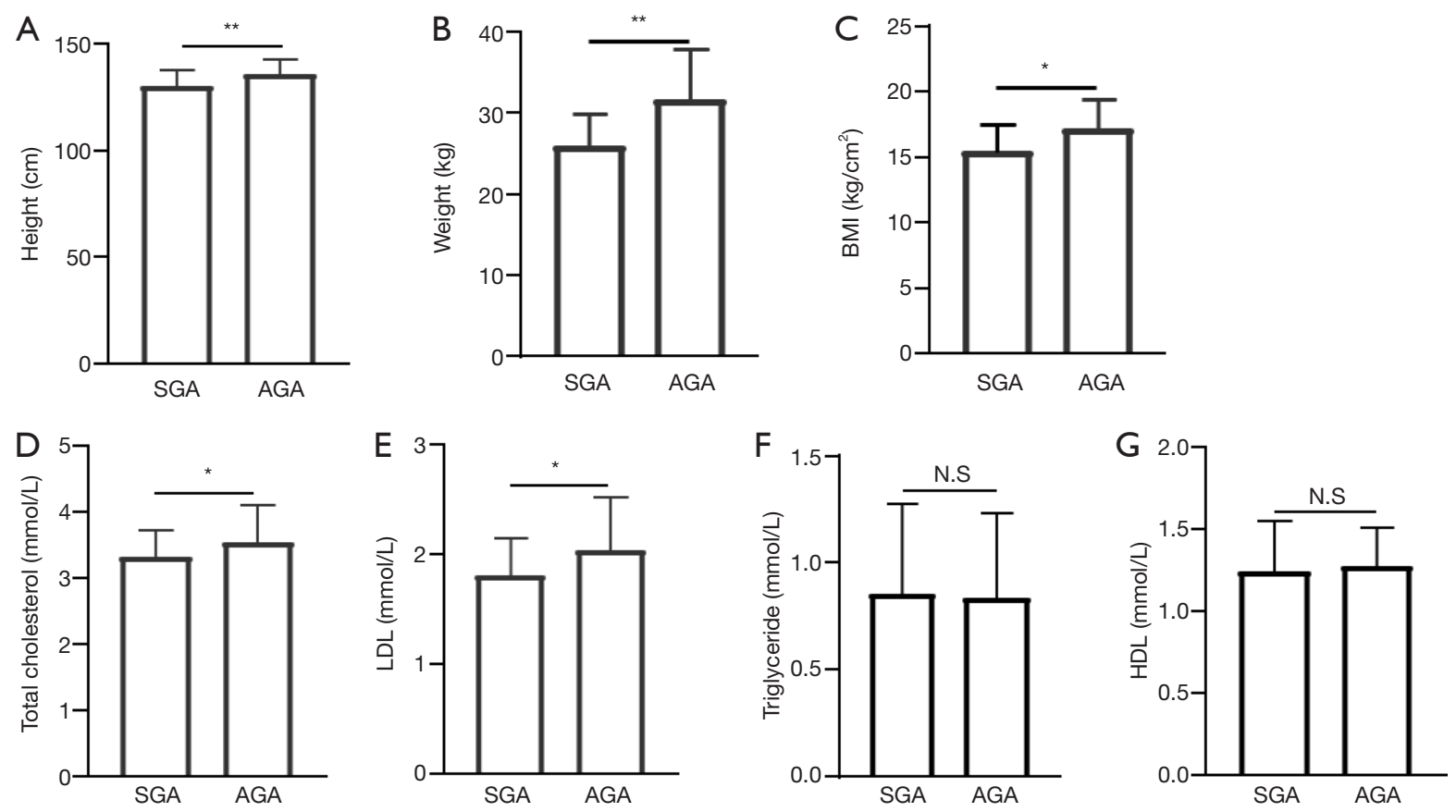

Figure 1 Comparison of physical indicators between the two groups; (A) height of the two groups; (B) weight of the two groups; (C) BMI of the two groups; (D) cholesterol levels in the two groups; (E) LDL levels in the two groups; (F) triglyceride levels in the two groups; (G) HDL levels in the two groups. *, $\mathrm{P}<0.05$; **, $\mathrm{P}<0.01$. N.S, no significance.

of follicle stimulating hormone (FSH) and luteinizing hormone ( $\mathrm{LH})$ were measured by ICMAS before the injection (baseline value) and 30, 60, 90, and 120 minutes after the injection. HPGA function starts, peak $\mathrm{LH}>5.0 \mu / \mathrm{L}$ and peak LH /FSH >0.6. Accelerated linear growth: annual growth rate is higher than normal. Gonadal development: pelvic b-ultrasound showed enlargement of the uterus and ovary and multiple follicles with a diameter of $>4 \mathrm{~mm}$ could be seen in the ovary.

\section{Statistical analysis}

SPSS 20.0 was used to analyze the data. Continuous data were expressed as mean $\pm \mathrm{SD}$. $T$-test was used for comparison between groups. The non-normally distributed continuous data were compared by non-parametric test. $\mathrm{P}<0.05$ was considered statistically significant.

\section{Results}

\section{General information about the girls}

According to the inclusion and exclusion criterions,
508 cases were excluded. At the end, a total of 322 girls with ICPP were divided into the SGA group and AGA group. In the SGA group, 18 patients $(5.5 \%)$ had an average age of $(8.23 \pm 0.91)$ years and 304 patients $(94.5 \%)$ in the AGA group and the mean age was $(8.26 \pm 1.08)$ years. The age was similar between the two groups $(\mathrm{t}=0.139, \mathrm{P}>0.05)$. The bone age of the two groups exceeded the actual age of more than 1 year. The bone age and the development of Tanner stage between the two groups was similar $(\mathrm{t}=1.088, \mathrm{P}>0.05)$.

\section{Comparison of physical indicators}

The average height of the AGA group was $(135.00 \pm 7.63)$ $\mathrm{cm}$, which was higher than the average height of the SGA group $(129.44 \pm 8.06 \mathrm{~cm} ; \mathrm{t}=2.996, \mathrm{P}<0.05)$. The average body weight and BMI of the AGA group were higher than those of the SGA group $(25.83 \pm 4.16$ vs. $31.50 \pm 6.31 \mathrm{~kg}$, $15.40 \pm 2.08 \mathrm{~kg} / \mathrm{cm}^{2}$ vs. $\left.17.16 \pm 2.31 \mathrm{~kg} / \mathrm{cm}^{2}\right)(\mathrm{t}=5.43, \mathrm{P}=0$; $\mathrm{t}=3.148, \mathrm{P}<0.05)$ (Figure $1 A, B, C)$.

\section{Comparison of sex hormones and adrenal androgens levels}

The level of LH/FSH, estradiol, testosterone, DHEAS and 
Table 1 Comparison of sex hormones and adrenal androgens levels between the two groups $(\bar{x} \pm s)$

\begin{tabular}{lccccc}
\hline Group & LH/FSH & $\mathrm{E}_{2}(\mathrm{pg} / \mathrm{mL})$ & $\mathrm{T}(\mathrm{ng} / \mathrm{mL})$ & DHEA $(\mathrm{ng} / \mathrm{mL})$ & Androstenedione $(\mathrm{ng} / \mathrm{mL})$ \\
\hline AGA & $0.29 \pm 0.46$ & $27.10 \pm 22.31$ & $0.35 \pm 0.40$ & $1172.90 \pm 806.63$ & $2.38 \pm 1.86$ \\
SGA & $0.21 \pm 0.19$ & $18.38 \pm 15.23$ & $0.28 \pm 0.27$ & $1050.35 \pm 616.64$ & $1.91 \pm 1.36$ \\
$\mathrm{t}$ & 0.770 & 1.636 & 0.782 & 0.633 & 1.055 \\
$\mathrm{P}$ & 0.44 & 0.1 & 0.43 & 0.53 & 0.29 \\
\hline
\end{tabular}

AGA, Appropriate for gestational age; SGA, Small for gestational age; LH, luteinizing hormone; FSH, Follicle-stimulating hormone; DHEA, dehydroepiandrosterone.

Table 2 Comparison of fasting blood glucose and fasting insulin levels between the two groups $(\bar{x} \pm \mathrm{s})$

\begin{tabular}{lccc}
\hline Group & FPG $(\mathrm{mmol} / \mathrm{L})$ & FINS $(\mathrm{mlU} / \mathrm{L})$ & HOMA-IR \\
\hline SGA & $4.66 \pm 0.52$ & $5.65 \pm 3.68$ & $1.20 \pm 0.85$ \\
AGA & $4.73 \pm 0.44$ & $6.81 \pm 3.55$ & $1.44 \pm 0.78$ \\
$\mathrm{t}$ & 0.658 & 1.343 & 1.29 \\
$\mathrm{P}$ & 0.511 & 0.181 & 0.198 \\
\hline
\end{tabular}

AGA, Appropriate for gestational age; SGA, Small for gestational age; FPG, fasting plasma glucose; FINS, fasting insulin; HOMA$\mathrm{IR}$, homeostasis model assessment of insulin resistance.

androstenedione had no significant difference between the SGA group and AGA group $(\mathrm{P}>0.05$; Table 1).

\section{Comparison of fasting blood glucose, fasting insulin levels, and HOMA-IR}

The fasting blood glucose, insulin level, and homeostasis model assessment of insulin resistance (HOMA-IR) had no significant difference between the SGA group and AGA group $(\mathrm{P}>0.05)$. Blood glucose and insulin levels in the two groups were within the normal range (Table 2).

\section{Comparison of blood lipid levels}

The average level of triglycerides had no significant difference between two group $(\mathrm{t}=-0.189, \mathrm{P}>0.05)$. There was a statistically significant difference in total cholesterol and low-density lipoprotein (LDL) between the two groups $(\mathrm{t}=2.084, \mathrm{P}<0.05 ; \mathrm{t}=2.050, \mathrm{P}<0.05)$. There was no significant difference in high-density lipoprotein (HDL) between the two groups $(\mathrm{t}=0.465, \mathrm{P}>0.05)$. However, the blood lipid levels in both groups were within the normal range (Figure 1D,E,F,G).

\section{Discussion}

SGA girls were 5-7 times more likely to have a shorter stature than AGA girls (12-14). Similar studies have reported that the adult height of SGA girls is 1 SD lower than that of AGA girls and most of this difference has been reflected before puberty (15). However, in addition to the gaps at birth and the extent of childhood catch-up, adolescent development is also an important factor affecting the height of SGA girls. Short SGA girls not only gain less height before puberty but also gain less height during puberty (16). Although the accelerated bone age of SGA girls with ICPP occurs earlier, the height and age at the onset of puberty were important for the final height (17). The results of our study presented that the height of SGA girls was lower than AGA girls.

Studies have shown that BMI of SGA may be lower than that of AGA and lower BMI is suitable for SGA growth (18). As a result, the management guidelines have been changed all over the world. SGA girls and AGA girls have different growth patterns during the ICPP period. SGA girls' bone maturation is accelerated and the peak bone growth rate occurs in early adolescence and those whose epiphyseal line closes at an accelerated rate (19). The duration of adolescent development of SGA girls is short and the height increase in the adolescent development period is smaller than expected. Therefore, SGA girls are at a higher risk of being shorter than their target height when the height of the baseline is lower, the growth space is smaller, and the growth time is shorter in the SGA group. During puberty, sex hormone and adrenal hormone levels are often higher than they were before puberty. Our study found no difference in these hormone levels between SGA and AGA in children with CPP. This may be related to the fact that all the selected cases were CPP.

Previous studies reported that insulin resistance is an 
important pathological process of metabolic syndrome and may occur early in life $(20,21)$. Soto et al. tested 1-yearold children who were born with SGA and found that girls who achieved catch-up had insulin resistance, while those who did not catch-up had not insulin resistance (22). Other previous studies indicated that the catch-up of SGA girls during growth and development may cause adipose accumulation and insulin resistance, therefore, leading to metabolic syndrome in adulthood $(23,24)$.

In our study, the fasting blood glucose, triglyceride, HOMA-IR and HDL were similar between the two groups; LDL was lower than the SGA group but both are within the normal range. By analyzing the reasons for the differences with the above studies, it was found that all the subjects in the abovementioned study were obese girls. The height and BMI of SGA girls were lower than AGA girls which suggested that there was no difference in glycolipid metabolism between the two groups possibly because SGA girls did not achieve catch-up. In addition, the small sample size of the SGA group may also be a contributing factor. In a previous prospective case-control study, individuals with SGA at birth have greater insulin resistance and higher prevalence of metabolic syndrome than individuals born with AGA at birth. It indicates that insulin resistance can be extended to middle age in individuals with SGA $(25,26)$. In the SGA group, there may still be catch-up in the subsequent growth and development, with continued accumulation of adipose tissue and the transition from insulin sensitivity to insulin resistance, which may have an impact on the metabolism of blood glucose and blood lipid and, thus, develop into metabolic syndrome. However, we did not find any evidence of abnormal glucose and lipid metabolism in the SGA girls at a mean age of age 8.3, it is possible these findings would emerge as the girls progress through puberty.

Limitations. Firstly, this study was a retrospective study and not a randomized controlled trial. Secondly, this study was a single-center trial and the sample size was limited. Another multiple center trial with large sample size was needed in the future. Thirdly, the clinical follow-up was short, the state of growth and development, level of blood glucose, and lipid metabolism should be observed with a longer follow-up. Fourthly, in the SGA group, there were only 18 participants were included in our study. Because, due to the limited sample size, only 18 cases with SGA were included in this study. Another reason maybe due to the low incidence rate of SGA girls with central precocious puberty (CPP) in our country. Another large trial with more participants was still need in the future. Fifthly, the effectiveness of length or weight-for-length on the metabolic status remained unknown and should be further researched. Sixthly, variations between clinical decision makers, ultrasound and $\mathrm{X}$-ray examiners cannot be standardized that may influenced the evaluation. Seventhly, there were no data about the height and weight of their parents which should be further researched.

\section{Conclusions}

In conclusion, the height, weight, BMI, serum cholesterol and LDL of girls in SGA with CPP were significantly lower than that of those girls born AGA. Therefore, it is important to pay close attention to the growth and metabolic markers in SGA girls in spite of the normal blood glucose and lipid metabolism level in this study. Because, these indicators may contribute to early diagnosis of SGA girls with CPP.

\section{Acknowledgments}

We would like to thank Berwick DM for his help in polishing our paper.

Funding: The project of natural science funding in Hubei province (2018CFB425).

\section{Footnote}

Reporting Checklist: The authors have completed the STROBE reporting checklist. Available at: http://dx.doi. org/10.21037/tp-20-366

Data Sharing Statement: Available at http://dx.doi. org/10.21037/tp-20-366

Conflicts of Interest: All authors have completed the ICMJE uniform disclosure form (available at: http://dx.doi. org/10.21037/tp-20-366 ). The authors have no conflicts of interest to declare.

Ethical Statement: The authors are accountable for all aspects of the work in ensuring that questions related to the accuracy or integrity of any part of the work are appropriately investigated and resolved. This study was conducted in accordance with the declaration of Helsinki (as was revised in 2013). This study was conducted with 
approval from the Ethics Committee of Tongji Hospital, Tongji Medical College, Huazhong University of Science and Technology (No. TJ-IRB20191011). All participants had signed the informed consent. Written informed consent was obtained from the guardians of the participants.

Open Access Statement: This is an Open Access article distributed in accordance with the Creative Commons Attribution-NonCommercial-NoDerivs 4.0 International License (CC BY-NC-ND 4.0), which permits the noncommercial replication and distribution of the article with the strict proviso that no changes or edits are made and the original work is properly cited (including links to both the formal publication through the relevant DOI and the license). See: https://creativecommons.org/licenses/by-nc-nd/4.0/.

\section{References}

1. Partsch CJ, Sippell WG. Pathogenesis and epidemiology of precocious puberty. Effects of exogenous oestrogens. Hum Reprod Update 2001;7:292-302.

2. Chen M, Eugster EA. Central Precocious Puberty: Update on Diagnosis and Treatment. Paediatr Drugs 2015;17:273-81.

3. Verkauskiene R, Petraitiene I, Albertsson Wikland K. Puberty in children born small for gestational age. Horm Res Paediatr 2013;80:69-77.

4. Farello G, Altieri C, Cutini M, et al. Review of the Literature on Current Changes in the Timing of Pubertal Development and the Incomplete Forms of Early Puberty. Front Pediatr 2019;7:147.

5. Sultan C, Gaspari L, Maimoun L, et al. Disorders of puberty. Best Pract Res Clin Obstet Gynaecol 2018;48:62-89.

6. Shen ZZ, Wang YW, Ma S, et al. Risk factors for preterm birth, low birth weight and small for gestational age: a prospective cohort study. Zhonghua Liu Xing Bing Xue Za Zhi 2019;40:1125-9.

7. Lin YJ. Metabolic syndrome in children and adolescents born premature and small-for-gestational age: A scenario of Developmental Origins of Health and Disease (DOHaD). Pediatr Neonatol 2018;59:109-10.

8. Cho AY, Ko SY, Lee JH, et al. Relationship between final adult height and birth weight after gonadotropin-releasing hormone agonist treatment in girls with central precocious puberty. Ann Pediatr Endocrinol Metab 2020;25:24-30.

9. Huang YT, Lin HY, Wang CH, et al. Association of preterm birth and small for gestational age with metabolic outcomes in children and adolescents: A population-based cohort study from Taiwan. Pediatr Neonatol 2018;59:147-53.

10. Willemsen RH, Elleri D, Williams RM, et al. Pros and cons of GnRHa treatment for early puberty in girls. Nat Rev Endocrinol 2014;10:352-63.

11. Zhu L, Zhang R, Zhang S, et al. Chinese neonatal birth weight curve for different gestational age. Zhonghua Er Ke Za Zhi 2015;53:97-103.

12. Willemsen RH, Dunger DB. Normal Variation in Pubertal Timing: Genetic Determinants in Relation to Growth and Adiposity. Endocr Dev 2016;29:17-35.

13. Latronico AC, Brito VN, Carel JC. Causes, diagnosis, and treatment of central precocious puberty. Lancet Diabetes Endocrinol 2016;4:265-74.

14. van der Steen M, Hokken-Koelega AC. Growth and Metabolism in Children Born Small for Gestational Age. Endocrinol Metab Clin North Am 2016;45:283-94.

15. Campisi SC, Carbone SE, Zlotkin S. Catch-Up Growth in Full-Term Small for Gestational Age Infants: A Systematic Review. Adv Nutr 2019;10:104-11.

16. Vicens-Calvet E, Espadero RM, Carrascosa A, et al. Small for Gestational Age. Longitudinal study of the pubertal growth spurt in children born small for gestational age without postnatal catch-up growth. J Pediatr Endocrinol Metab 2002;15:381-8.

17. Deng HZ, Deng H, Su Z, et al. Insulin resistance and adiponectin levels are associated with height catch-up growth in pre-pubertal Chinese individuals born small for gestational age. Nutr Metab (Lond) 2012;9:107.

18. Shoji $\mathrm{H}$, Watanabe A, Awaji A, et al. Intrauterine growth restriction affects $\mathrm{z}$-scores of anthropometric parameters during the first 6 years in very low-birth-weight-children born at less than 30 weeks of gestation. J Dev Orig Health Dis 2020;11:44-8.

19. Lazar L, Pollak U, Kalter-Leibovici O, et al. Pubertal course of persistently short children born small for gestational age (SGA) compared with idiopathic short children born appropriate for gestational age (AGA). Eur J Endocrinol 2003;149:425-32.

20. Liu C, Wu B, Lin N, et al. Insulin resistance and its association with catch-up growth in Chinese children born small for gestational age. Obesity (Silver Spring) 2017;25:172-7.

21. Mericq V, Martinez-Aguayo A, Uauy R, et al. Long-term metabolic risk among children born premature or small for gestational age. Nat Rev Endocrinol 2017;13:50-62.

22. Soto N, Bazaes RA, Peña V, et al. Insulin sensitivity 
and secretion are related to catch-up growth in smallfor-gestational-age infants at age 1 year: results from a prospective cohort. J Clin Endocrinol Metab 2003;88:3645-50.

23. Cho WK, Suh BK. Catch-up growth and catch-up fat in children born small for gestational age. Korean J Pediatr 2016;59:1-7.

24. Stroescu R, Micle I, Bizerea T, et al. Metabolic monitoring of obese children born small for gestational age. Obes Res

Cite this article as: Yu X, Yang X, Zhao Y, Luo XP, Liang Y, Wu W, Ying YQ, Ye F, Hou L. A comparison of the growth status, level of blood glucose, and lipid metabolism in small for gestational age and appropriate for gestational age girls with central precocious puberty: a retrospective study. Transl Pediatr 2021;10(4):783-789. doi: 10.21037/tp-20-366
Clin Pract 2014;8:e592-8.

25. Argente J, Mehls O, Barrios V. Growth and body composition in very young SGA children. Pediatr Nephrol 2010;25:679-85.

26. Meas T, Deghmoun S, Alberti C, et al. Independent effects of weight gain and fetal programming on metabolic complications in adults born small for gestational age. Diabetologia 2010;53:907-13. 